\title{
Fixed point theorems for contractions of rational type in complete metric spaces
}

\section{Tomonari Suzuki}

Department of Basic Sciences, Faculty of Engineering, Kyushu Institute of Technology, Tobata, Kitakyushu 804-8550, Japan.

\author{
Communicated by R. Saadati
}

\begin{abstract}
Samet et al. in [S. Samet, C. Vetro, H. Yazidi, J. Nonlinear Sci. Appl., 6 (2013), 162-169] proved some fixed point theorem for contractions of rational type. In order to clarify the mathematical structure of contractions of rational type, we generalize this theorem in a general setting.
\end{abstract}

Keywords: Fixed point, contraction of rational type, complete metric space.

2010 MSC: $54 \mathrm{H} 25$.

(c)2018 All rights reserved.

\section{Introduction}

In 2013, Samet et al. proved the following interesting fixed point theorem.

Theorem 1.1 ([14, Theorem 2.1]). Let $(\mathrm{X}, \mathrm{d})$ be a complete metric space and let $\mathrm{T}$ be a mapping on $\mathrm{X}$. Assume that for any $\varepsilon>0$, there exists $\delta>0$ such that

$$
2 \varepsilon \leqslant d(y, T y) \frac{1+d(x, T x)}{1+d(x, y)}+d(x, y)<2 \varepsilon+\delta
$$

implies $\mathrm{d}(\mathrm{T} x, \mathrm{Ty})<\varepsilon$. Then $\mathrm{T}$ has a unique fixed point $z$. Moreover $\left\{\mathrm{T}^{\mathrm{n}} \mathrm{x}\right\}$ converges to $z$ for all $\mathrm{x} \in \mathrm{X}$.

We recently call such a mapping $T$ a contraction of rational type. The idea of Theorem 1.1 comes from Dass and Gupta [4] and Meir and Keeler [12].

Theorem 1.2 ([4, Theorem 1$])$. Let $(\mathrm{X}, \mathrm{d})$ be a complete metric space and let $\mathrm{T}$ be a mapping on $\mathrm{X}$. Assume that there exist $\alpha, \beta \in(0,1)$ satisfying $\alpha+\beta<1$ and

$$
d(T x, T y) \leqslant \alpha d(y, T y) \frac{1+d(x, T x)}{1+d(x, y)}+\beta d(x, y)
$$

for all $x, y \in X$. Then $T$ has a unique fixed point $z$. Moreover $\left\{\mathrm{T}^{\mathrm{n}} \mathrm{x}\right\}$ converges to $z$ for all $\mathrm{x} \in \mathrm{X}$.

Email address: suzuki-t@mns.kyutech.ac.jp (Tomonari Suzuki)

doi: $10.22436 /$ jnsa.011.01.08

Received: 2017-08-17 Revised: 2017-11-06 Accepted: 2017-11-08 
Theorem 1.3 ([12]). Let $(\mathrm{X}, \mathrm{d})$ be a complete metric space and let $\mathrm{T}$ be a mapping on $\mathrm{X}$. Assume that for any $\varepsilon>0$, there exists $\delta>0$ such that

$$
\mathrm{d}(\mathrm{x}, \mathrm{y})<\varepsilon+\delta \text { implies } \mathrm{d}(\mathrm{T} x, \mathrm{~T} y)<\varepsilon
$$

for all $x, y \in X$. Then $T$ has a unique fixed point $z$. Moreover $\left\{\mathrm{T}^{\mathrm{n}} \mathrm{x}\right\}$ converges to $z$ for all $\mathrm{x} \in \mathrm{X}$.

We note that Theorems 1.2 and 1.3 are generalizations of the Banach contraction principle [1, 2]. However, unfortunately, Theorem 1.1 is not a generalization of Theorems 1.2 and 1.3. Motivated by this fact, in this paper, we study the mathematical structure of contractions of rational type. Also we modify Theorem 1.1 in order to become a generalization of Theorems 1.2 and 1.3.

\section{Preliminaries}

Throughout this paper we denote by $\mathbb{N}$ the set of all positive integers. For an arbitrary set $X$, we define $X^{(2)}$ by

$$
X^{(2)}=\{(x, y) \in X \times X: x \neq y\} .
$$

In this section, we give some preliminaries.

Let $(X, d)$ be a metric space and let $T$ be a mapping on $X$. Define functions $K$ and $L$ from $X \times X$ into $[0, \infty)$ by

$$
\begin{aligned}
& K(x, y)=\max \left\{d(x, y), \frac{d(x, T y)+d(T x, y)}{2}, \frac{d(x, T x)+d(y, T y)}{2}\right\}, \\
& L(x, y)=\max \left\{d(x, y), \frac{d(x, T y)+d(T x, y)}{2}, d(x, T x), d(y, T y)\right\} .
\end{aligned}
$$

Let $p$ be a function from $X^{(2)}$ into $[0, \infty)$ and let $c \in[0,1)$. We introduce the following conditions.

$(P 1: p) x \neq y$ and $d(x, T x) \leqslant d(x, y)$ imply $p(x, y) \leqslant L(x, y)$.

$(P 2: p, c) x \neq y_{n}, \lim _{n} d\left(x, y_{n}\right)=0$ and $\lim _{n} d\left(y_{n}, T y_{n}\right)=0$ imply

$$
\limsup _{n \rightarrow \infty} p\left(x, y_{n}\right) \leqslant c d(x, T x) \text {. }
$$

The following lemma plays an important role in this paper, though its proof is easy.

Lemma 2.1. Let $p_{1}$ and $p_{2}$ be functions from $X^{(2)}$ into $[0, \infty)$ and let $\left\{q_{i}: i \in I\right\}$ be a family of functions from $X^{(2)}$ into $[0, \infty)$. Let $\mathrm{c} \in[0,1)$. Define functions $\mathrm{p}_{3}, \mathrm{p}_{4}$, and $\mathrm{p}_{5}$ by

$$
p_{3}(x, y)=\sup \left\{q_{i}(x, y): i \in I\right\}, \quad p_{4}(x, y)=\max \left\{p_{1}(x, y), p_{2}(x, y)\right\}, \quad p_{5}(x, y)=\alpha p_{1}(x, y)+\beta p_{2}(x, y)
$$

for $(x, y) \in X^{(2)}$, where $\alpha, \beta \in(0,1)$ with $\alpha+\beta=1$. Then the following hold.

(i) If $\mathrm{p}_{1} \leqslant \mathrm{p}_{2}$ holds and $\mathrm{p}_{2}$ satisfies (P1: $\left.\mathrm{p}_{2}\right)$, then $\mathrm{p}_{1}$ also satisfies (P1: $\left.\mathrm{p}_{1}\right)$.

(ii) If $\mathrm{p}_{1} \leqslant \mathrm{p}_{2}$ holds and $\mathrm{p}_{2}$ satisfies (P2: $\left.\mathrm{p}_{2}, \mathrm{c}\right)$, then $\mathrm{p}_{1}$ also satisfies (P2: $\left.\mathrm{p}_{1}, \mathrm{c}\right)$.

(iii) If $\mathrm{p}_{3}(\mathrm{x}, \mathrm{y})<\infty$ holds for $(\mathrm{x}, \mathrm{y}) \in \mathrm{X}^{(2)}$ and $\mathrm{q}_{\mathrm{i}}$ satisfies $\left(\mathrm{P} 1: \mathrm{q}_{i}\right)$ for $\mathrm{i} \in \mathrm{I}$, then $\mathrm{p}_{3}$ also satisfies (P1: $\left.\mathrm{p}_{3}\right)$.

(iv) If $\mathrm{p}_{1}$ and $\mathrm{p}_{2}$ satisfy $\left(\mathrm{P} 2: \mathrm{p}_{1}, \mathrm{c}\right)$ and $\left(\mathrm{P} 2: \mathrm{p}_{2}, \mathrm{c}\right)$, then $\mathrm{p}_{4}$ also satisfies $\left(\mathrm{P} 2: \mathrm{p}_{4}, \mathrm{c}\right)$.

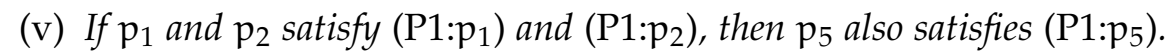

(vi) If $\mathrm{p}_{1}$ and $\mathrm{p}_{2}$ satisfy $\left(\mathrm{P} 2: \mathrm{p}_{1}, \mathrm{c}\right)$ and $\left(\mathrm{P} 2: \mathrm{p}_{2}, \mathrm{c}\right)$, then $\mathrm{p}_{5}$ also satisfies $\left(\mathrm{P} 2: \mathrm{p}_{5}, \mathrm{c}\right)$.

Proof. From the definition of the conditions (P1) and (P2), we can easily prove (i)-(iv). (v) follows from (i) and (iii). (vi) follows from (ii) and (iv).

We give examples which satisfy (P1) and (P2). 
Example 2.2. The following hold.

(i) (P1:d) and (P2:d,0) hold.

(ii) (P1:K) and (P2:K, 1/2) hold.

(iii) (P1:L) holds.

Proof. Since $d \leqslant K \leqslant L$ and $L \leqslant L$ hold, we obtain (P1:d), (P1:K), and (P1:L). (P2:d, 0 ) obviously holds. If $x \neq y_{n}, \lim _{n} d\left(x, y_{n}\right)=0$ and $\lim _{n} d\left(y_{n}, T y_{n}\right)=0$ hold, then we have

$$
\limsup _{n \rightarrow \infty} K\left(x, y_{n}\right)=(1 / 2) d(T x, x),
$$

thus, (P2:K, 1/2) holds.

Example 2.3. Define functions $p_{1}-p_{6}$ from $X^{(2)}$ into $[0, \infty)$ by

$$
\begin{array}{ll}
p_{1}(x, y)=d(y, T y) \frac{1+d(x, T x)}{1+d(x, y)}, & p_{2}(x, y)=\max \left\{p_{1}(x, y), d(x, y)\right\}, \\
p_{3}(x, y)=(1 / 2)\left(p_{1}(x, y)+d(x, y)\right), & p_{4}(x, y)=\frac{d(x, T x) d(y, T y)}{d(x, y)}, \\
p_{5}(x, y)=\max \left\{p_{1}(x, y), p_{4}(x, y), d(x, y)\right\}, & p_{6}(x, y)=(1 / 3)\left(p_{1}(x, y)+p_{4}(x, y)+d(x, y)\right) .
\end{array}
$$

Then (P1: $\left.p_{1}\right),\left(P 2: p_{1}, 0\right),\left(P 1: p_{2}\right),\left(P 2: p_{2}, 0\right),\left(P 1: p_{3}\right),\left(P 2: p_{3}, 0\right),\left(P 1: p_{4}\right),\left(P 1: p_{5}\right)$, and (P1:p 6$)$ hold.

Proof. We assume $x \neq y$ and $d(x, T x) \leqslant d(x, y)$. Then we have

$$
p_{1}(x, y) \leqslant d(y, T y) \leqslant L(x, y)
$$

Therefore $\left(\mathrm{P} 1: \mathrm{p}_{1}\right)$ holds. Similarly we can prove $\left(\mathrm{P} 1: \mathrm{p}_{4}\right)$.

We assume $x \neq y_{n}, \lim _{n} d\left(x, y_{n}\right)=0$ and $\lim _{n} d\left(y_{n}, T y_{n}\right)=0$. Then we have

$$
\limsup _{n \rightarrow \infty} p_{1}\left(x, y_{n}\right) \leqslant \limsup _{n \rightarrow \infty} d\left(y_{n}, T y_{n}\right)(1+d(x, T x))=0 .
$$

Therefore $\left(\mathrm{P} 2: \mathrm{p}_{1}, 0\right)$ holds.

So by Lemma 2.1 (iii) and (iv) and Example 2.2 (i), we obtain $\left(\mathrm{P} 1: \mathrm{p}_{2}\right)$ and (P2: $\left.\mathrm{p}_{2}, 0\right)$. By Lemma 2.1 (v) and (vi), we obtain $\left(\mathrm{P} 1: \mathrm{p}_{3}\right)$ and $\left(\mathrm{P} 2: \mathrm{p}_{3}, 0\right)$. (P1: $\left.\mathrm{p}_{5}\right)$ and $\left(\mathrm{P} 1: \mathrm{p}_{6}\right)$ follow from Lemma 2.1 (iii) and (v), respectively.

We can easily prove the following lemma. However, we give a proof because Lemma 2.4 is important in this paper.

Lemma 2.4. Let $(\mathrm{X}, \mathrm{d})$ be a metric space and let $\mathrm{T}$ be a mapping on $\mathrm{X}$. Let $\mathrm{p}$ be a function from $\mathrm{X} \times \mathrm{X}$ into $[0, \infty)$ such that $\mathrm{p}(\mathrm{x}, \mathrm{y})=0$ implies $\mathrm{d}(\mathrm{T} x, \mathrm{Ty})=0$. Then the following are equivalent.

(i) For any $\varepsilon>0$, there exists $\delta>0$ such that

$$
x \neq y, \varepsilon \leqslant p(x, y)<\varepsilon+\delta \quad \text { imply } \quad d(T x, T y)<\varepsilon .
$$

(ii) For any $\varepsilon>0$, there exists $\delta>0$ such that

$$
x \neq y, p(x, y)<\varepsilon+\delta \quad \text { imply } \quad \mathrm{d}(\mathrm{T} x, \mathrm{Ty})<\varepsilon .
$$

(iii) For any $\varepsilon>0$, there exists $\delta>0$ such that

$$
\varepsilon \leqslant p(x, y)<\varepsilon+\delta \quad \text { implies } \mathrm{d}(\mathrm{T} x, \mathrm{Ty})<\varepsilon .
$$


(iv) For any $\varepsilon>0$, there exists $\delta>0$ such that

$$
p(x, y)<\varepsilon+\delta \text { implies } \mathrm{d}(\mathrm{T} x, \mathrm{Ty})<\varepsilon .
$$

Remark 2.5. We note that the values of $p(x, x)$ have no influence in this context. Indeed, if $p$ satisfies (i), then $q$ also satisfies (i) provided $p(x, y)=q(x, y)$ holds for all $(x, y) \in X^{(2)}$.

Proof. (iv) $\Rightarrow$ (ii) $\Rightarrow$ (i) obviously holds. It is also obvious that (iv) $\Rightarrow$ (iii) $\Rightarrow$ (i) holds. Let us prove (i) $\Rightarrow$ (iv). Fix $\varepsilon>0$ and choose $\delta>0$ appearing in (i). Fix $x, y \in X$ with $p(x, y)<\varepsilon+\delta$. We consider the following four cases.
(a) $x \neq y$ and $\varepsilon \leqslant p(x, y)$.
(b) $x \neq y$ and $0<p(x, y)<\varepsilon$.
(c) $x \neq y$ and $p(x, y)=0$.
(d) $x=y$.

In the case of $(a), d(T x, T y)<\varepsilon$ obviously holds. In the cases of $(c)$ and $(d)$, we have $d(T x, T y)=0<\varepsilon$. In the case of $(b)$, we put $\varepsilon_{2}:=p(x, y)>0$. Then there exists $\delta_{2}>0$ such that

$$
u \neq v, \varepsilon \leqslant p(u, v)<\varepsilon_{2}+\delta_{2} \quad \text { imply } \quad d(T u, T v)<\varepsilon_{2} .
$$

Since $\varepsilon_{2} \leqslant p(x, y)<\varepsilon_{2}+\delta_{2}$ holds, we have $d(T x, T y)<\varepsilon_{2}<\varepsilon$.

\section{Fixed point theorems}

In this section, we prove fixed point theorems.

Theorem 3.1. Let $(\mathrm{X}, \mathrm{d})$ be a complete metric space and let $\mathrm{T}$ be a mapping on $\mathrm{X}$. Let $\mathrm{p}$ be a function from $\mathrm{X}^{(2)}$ into $[0, \infty)$ satisfying (P1:p) and (P2:p, c) for some $\mathrm{c} \in[0,1)$. Assume the following.

(i) For any $\varepsilon>0$, there exists $\delta(\varepsilon)>0$ such that $x \neq y$ and $p(x, y)<\varepsilon+\delta(\varepsilon)$ imply $d(T x, T y) \leqslant \varepsilon$.

(ii) $x \neq y$ and $p(x, y)>0$ imply $d(T x, T y)<p(x, y)$.

Then $\mathrm{T}$ has a unique fixed point $z$. Moreover $\left\{\mathrm{T}^{\mathrm{n}} \mathrm{x}\right\}$ converges to $z$ for all $\mathrm{x} \in \mathrm{X}$.

Proof. Without loss of generality, we may assume $\delta(\varepsilon)<\varepsilon$.

We will show

$$
p(x, y)=0 \Rightarrow T x=T y .
$$

Let $x, y \in X$ satisfy $p(x, y)=0$. In the case where $x=y$, it is obvious that $T x=T y$ holds. In the other case, where $x \neq y$, from (i), we have $d(T x, T y) \leqslant \varepsilon$ for any $\varepsilon>0$. Since $\varepsilon>0$ is arbitrary, we obtain $T x=T y$. We have shown (3.1). Next we show

$$
x \neq T x \wedge p(x, T x)>0 \Rightarrow d\left(T x, T^{2} x\right)<p(x, T x) \leqslant L(x, T x)=d(x, T x) .
$$

Let $x \in X$ satisfy $x \neq T x$ and $p(x, T x)>0$. Then we have by (P1:p),

$$
\begin{aligned}
p(x, T x) \leqslant L(x, T x) & =\max \left\{d(x, T x), \frac{d\left(x, T^{2} x\right)}{2}, d\left(T x, T^{2} x\right)\right\} \\
& =\max \left\{d(x, T x), \frac{d(x, T x)+d\left(T x, T^{2} x\right)}{2}, d\left(T x, T^{2} x\right)\right\} \\
& =\max \left\{d(x, T x), d\left(T x, T^{2} x\right)\right\} .
\end{aligned}
$$

If $d(x, T x)<d\left(T x, T^{2} x\right)$ holds, then we have by (ii)

$$
\mathrm{d}\left(\mathrm{T} x, \mathrm{~T}^{2} x\right)<\mathrm{p}(x, \mathrm{~T} x) \leqslant \max \left\{\mathrm{d}(x, \mathrm{~T} x), \mathrm{d}\left(\mathrm{T} x, \mathrm{~T}^{2} x\right)\right\}=\mathrm{d}\left(\mathrm{T} x, \mathrm{~T}^{2} x\right),
$$

which implies a contradiction. So we have $p(x, T x) \leqslant L(x, T x)=d(x, T x)$. By (ii), we obtain (3.2).

Fix $u \in X$. We consider the following two cases. 
(a) $T^{v} u=T^{v+1} u$ for some $v \in \mathbb{N}$.

(b) $T^{n} u \neq T^{n+1} u$ for any $n \in \mathbb{N}$.

In the case of (a), we put $z=T^{\vee} u$. Then it is obvious that $z$ is a fixed point of $T$ and $\left\{T^{n} u\right\}$ converges to $z$.

In the case of (b), noting (3.1), we have $p\left(T^{n} \mathfrak{u}, T^{n+1} \mathfrak{u}\right)>0$ for any $n \in \mathbb{N}$. So by (3.2), $\left\{d\left(T^{n} \mathfrak{u}, T^{n+1} \mathfrak{u}\right)\right\}$ is strictly decreasing. So we note that $T^{n} \mathfrak{u}(n \in \mathbb{N})$ are all different. Also, $\left\{d\left(T^{n} u, T^{n+1} \mathfrak{u}\right)\right\}$ converges to some $\alpha \in[0, \infty)$. We note $\alpha<d\left(T^{n} u, T^{n+1} \mathfrak{u}\right)$ for any $n \in \mathbb{N}$. Arguing by contradiction, we assume $\alpha>0$. Then for sufficiently large $n \in \mathbb{N}$, we have by (3.2)

$$
p\left(T^{n} u, T^{n+1} u\right) \leqslant d\left(T^{n} u, T^{n+1} u\right)<\alpha+\delta(\alpha)
$$

and hence

$$
\alpha<d\left(T^{n+1} u, T^{n+2} u\right) \leqslant \alpha,
$$

which implies a contradiction. Therefore we have shown

$$
\lim _{n \rightarrow \infty} d\left(T^{n} u, T^{n+1} u\right)=0 .
$$

In order to show that $\left\{T^{n} \mathfrak{u}\right\}$ is a Cauchy sequence, we fix $\varepsilon>0$. By (3.3), we can choose $\ell \in \mathbb{N}$ satisfying

$$
\mathrm{d}\left(\mathrm{T}^{\ell} \mathrm{u}, \mathrm{T}^{\ell+1} \mathfrak{u}\right)<\delta(\varepsilon) / 2 \text {. }
$$

By induction, we will show

$$
\mathrm{d}\left(\mathrm{T}^{\ell} \mathrm{u}, \mathrm{T}^{\ell+\mathrm{k}} \mathrm{u}\right)<\varepsilon+\delta(\varepsilon) / 2
$$

for any $k \in \mathbb{N}$. It is obvious that (3.4) holds for $k:=1$. We assume that (3.4) holds for some $k \in \mathbb{N}$. Then we consider the following two cases.

- $d\left(T^{\ell} u, T^{\ell+k} u\right) \leqslant \varepsilon$.

- $d\left(T^{\ell} u, T^{\ell+k} u\right)>\varepsilon$.

In the first case, we have by (3.2)

$$
d\left(T^{\ell} u, T^{\ell+k+1} u\right) \leqslant d\left(T^{\ell} u, T^{\ell+k} u\right)+d\left(T^{\ell+k} u, T^{\ell+k+1} u\right)<\varepsilon+d\left(T^{\ell} u, T^{\ell+1} u\right)<\varepsilon+\delta(\varepsilon) / 2 .
$$

In the second case, we have

$$
\mathrm{d}\left(\mathrm{T}^{\ell} \mathrm{u}, \mathrm{T}^{\ell+1} \mathrm{u}\right)<\delta(\varepsilon) / 2<\delta(\varepsilon)<\varepsilon<\mathrm{d}\left(\mathrm{T}^{\ell} \mathrm{u}, \mathrm{T}^{\ell+k} \mathrm{u}\right)
$$

and hence $p\left(T^{\ell} u, T^{\ell+k} u\right) \leqslant L\left(T^{\ell} u, T^{\ell+k} u\right)$ by (P1:p). We also have

$$
\begin{aligned}
d\left(T^{\ell} u, T^{\ell+k+1} u\right)+d\left(T^{\ell+1} u, T^{\ell+k} u\right) \leqslant & d\left(T^{\ell} u, T^{\ell+k} u\right)+d\left(T^{\ell+k} u, T^{\ell+k+1} u\right) \\
& +d\left(T^{\ell+1} u, T^{\ell} u\right)+d\left(T^{\ell} u, T^{\ell+k} u\right)<2 \varepsilon+2 \delta(\varepsilon)
\end{aligned}
$$

and hence

$$
p\left(T^{\ell} u, T^{\ell+k} u\right) \leqslant L\left(T^{\ell} u, T^{\ell+k} u\right)<\max \{\varepsilon+\delta(\varepsilon) / 2, \varepsilon+\delta(\varepsilon), \delta(\varepsilon) / 2, \delta(\varepsilon) / 2\}=\varepsilon+\delta(\varepsilon) .
$$

So we have by (i)

$$
d\left(T^{\ell+1} u, T^{\ell+k+1} u\right) \leqslant \varepsilon
$$

and hence

$$
d\left(T^{\ell} u, T^{\ell+k+1} \mathfrak{u}\right) \leqslant d\left(T^{\ell} u, T^{\ell+1} \mathfrak{u}\right)+d\left(T^{\ell+1} u, T^{\ell+k+1} \mathfrak{u}\right)<\delta(\varepsilon) / 2+\varepsilon .
$$

We have shown (3.4) for $k:=k+1$ in both cases. By induction, we have (3.4) for any $k \in \mathbb{N}$. Since $\varepsilon>0$ is arbitrary and $\varepsilon+\delta(\varepsilon) / 2<(3 / 2) \varepsilon$ holds, we obtain

$$
\lim _{n \rightarrow \infty} \sup \left\{d\left(T^{n} u, T^{m} u\right): m>n\right\}=0,
$$

which implies that $\left\{T^{n} u\right\}$ is Cauchy. Since $X$ is complete, $\left\{T^{n} u\right\}$ converges to some $z \in X$. Since $T^{n} u$ 
( $n \in \mathbb{N}$ ) are all different, we note $z \neq T^{n} u$ for sufficiently large $n \in \mathbb{N}$. Since $\lim _{n} d\left(z, T^{n} u\right)=0$ and $\lim _{n} d\left(T^{n} u, T^{n+1} \mathfrak{u}\right)=0$ hold, we have by $(P 2: p, c)$

$$
\limsup _{n \rightarrow \infty} p\left(z, T^{n} u\right) \leqslant c d(z, T z) .
$$

We have by (ii) and (3.1)

$$
d(T z, z)=\lim _{n \rightarrow \infty} d\left(T z, T^{n} u\right) \leqslant \limsup _{n \rightarrow \infty} p\left(z, T^{n-1} u\right) \leqslant c d(z, T z)
$$

and hence $z$ is a fixed point of T.

Therefore we have shown that $\left\{T^{n} u\right\}$ converges to a fixed point $z$ of $T$ in the cases of (a) and (b). Let $w \in X$ be a distinct fixed point of T. Then if $p(z, w)=0$ holds, then we have by (3.1)

$$
0<\mathrm{d}(z, w)=\mathrm{d}(\mathrm{T} z, \mathrm{~T} w)=0,
$$

which implies a contradiction. Therefore $p(z, w)>0$ holds. From (P1:p), we have

$$
p(z, w) \leqslant \mathrm{L}(z, w)=\mathrm{d}(z, w) .
$$

We have by (ii)

$$
\mathrm{d}(z, w)=\mathrm{d}(\mathrm{T} z, \mathrm{~T} w)<p(z, w) \leqslant \mathrm{d}(z, w),
$$

which implies a contradiction. Therefore the fixed point $z$ is unique.

Theorem 3.2. Let $(\mathrm{X}, \mathrm{d})$ be a complete metric space and let $\mathrm{T}$ be a continuous mapping on $\mathrm{X}$. Let $\mathrm{p}$ be a function from $\mathrm{X}^{(2)}$ into $[0, \infty)$ satisfying $(\mathrm{P} 1: \mathrm{p})$. Assume (i) and (ii) of Theorem 3.1. Then $\mathrm{T}$ has a unique fixed point $z$. Moreover $\left\{\mathrm{T}^{\mathrm{n}} \mathrm{x}\right\}$ converges to $z$ for all $\mathrm{x} \in \mathrm{X}$.

Proof. Fix $u \in X$. Then as in the proof of Theorem 3.1, we can prove that $\left\{T^{n} u\right\}$ converges to some $z \in X$. Since $T$ is continuous, we have

$$
\mathrm{T} z=\mathrm{T}\left(\lim _{n \rightarrow \infty} \mathrm{T}^{\mathrm{n}} \mathrm{u}\right)=\lim _{n \rightarrow \infty} \mathrm{T} \circ \mathrm{T}^{\mathrm{n}} \mathrm{u}=z,
$$

thus, $z$ is a fixed point of T. We can prove the uniqueness of the fixed point as in the proof of Theorem 3.1 .

\section{Contractive condition}

In this section, we discuss the contractive condition on Theorems 3.1 and 3.2.

Let $(X, d)$ be a metric space and let $T$ be a mapping on $X$. Then using subsets $Q$ of $[0, \infty)^{2}$ defined by

$$
Q=\{(d(x, y), d(T x, T y)): x, y \in X\},
$$

Hegedüs and Szilágyi in [6] studied some contractive conditions. See $[8,15]$ and references therein. The merit of the usage of $Q$ is to hide the mapping $T$ and the inequality, in particular, the right hand side of the inequality. In other words, we can concentrate only on contractive conditions.

Definition 4.1. Let $\mathrm{Q}$ be a subset of $[0, \infty)^{2}$. Then $\mathrm{Q}$ is said to be $C J M$ if the following hold.

(i) For any $\varepsilon>0$, there exists $\delta>0$ such that $u \leqslant \varepsilon$ holds for any $(t, u) \in Q$ with $t<\varepsilon+\delta$.

(ii) $u<t$ holds for any $(t, u) \in Q$ with $t>0$.

It is obvious that Q defined by (4.1) is CJM iff T satisfies (i) and (ii) of Theorem 5.1 below. We prove the following. 
Lemma 4.2. Let $(\mathrm{X}, \mathrm{d})$ be a metric space and let $\mathrm{T}$ be a mapping on $\mathrm{X}$. Let $\mathrm{p}$ be a function from $\mathrm{X} \times \mathrm{X}$ into $[0, \infty)$. Then (c) $\Rightarrow$ (b) $\Leftrightarrow$ (ii) of Theorem 3.1 holds.

(b) $p(x, y)>0$ implies $d(T x, T y)<p(x, y)$.

(c) $x \neq y$ implies $d(T x, T y)<p(x, y)$.

Moreover, we assume $\mathrm{p}(\mathrm{x}, \mathrm{y})=0 \Rightarrow \mathrm{x}=\mathrm{y}$ additionally, then (c), (b), and (ii) of Theorem 3.1 are equivalent.

Proof. It is obvious that the disjunction of (b) and (c) implies (ii) of Theorem 3.1.

Let us prove that (ii) of Theorem 3.1 implies (b). Fix $x, y \in X$ with $p(x, y)>0$. In the case where $x \neq y$, we have $d(T x, T y)<p(x, y)$ by (ii) of Theorem 3.1. In the other case, where $x=y$, we have $d(T x, T y)=0<p(x, y)$. Thus, (b) holds.

Now we assume $p(x, y)=0 \Rightarrow x=y$ additionally. Then $x \neq y$ implies $p(x, y)>0$ holds. So we can prove $(b) \Rightarrow(c)$.

Lemma 4.3. Let $(\mathrm{X}, \mathrm{d})$ be a metric space and let $\mathrm{T}$ be a mapping on $\mathrm{X}$. Let $\mathrm{p}$ be a function from $\mathrm{X} \times \mathrm{X}$ into $[0, \infty)$. Then the conjunction of (i) and (ii) of Theorem 3.1 is equivalent to the conjunction of the following (a) and (b).

(a) For any $\varepsilon>0$, there exists $\delta>0$ such that

$$
p(x, y)<\varepsilon+\delta \quad \text { implies } \quad d(T x, T y) \leqslant \varepsilon .
$$

(b) $p(x, y)>0$ implies $d(T x, T y)<p(x, y)$.

Remark 4.4. We note that the values of $p(x, x)$ have no influence in this context.

Proof. By Lemma 4.2, we have proved that (b) is equivalent to (ii) of Theorem 3.1. It is obvious that (a) implies (i) of Theorem 3.1. As in the proof of Lemma 4.2, we can prove that (i) of Theorem 3.1 implies (a).

By Lemma 4.3, we can prove the following.

Proposition 4.5. Let $(\mathrm{X}, \mathrm{d})$ be a metric space and let $\mathrm{T}$ be a mapping on $\mathrm{X}$. Let $\mathrm{p}$ be a function from $\mathrm{X} \times \mathrm{X}$ into $[0, \infty)$. Define a subset $\mathrm{R}$ of $[0, \infty)^{2}$ by

$$
R=\{(p(x, y), d(T x, T y)): x, y \in X\} .
$$

Then the following are equivalent.

(i) T satisfies (i) and (ii) of Theorem 3.1.

(ii) $\mathrm{R}$ is CJM.

By Lemma 4.3 again, we obtain the following.

Corollary 4.6. Let $(\mathrm{X}, \mathrm{d})$ be a complete metric space and let $\mathrm{T}$ be a mapping on $\mathrm{X}$. Let $\mathrm{p}$ be a function from $\mathrm{X} \times \mathrm{X}$ into $[0, \infty)$ satisfying $(\mathrm{P} 1: \mathrm{p})$ and $(\mathrm{P} 2: \mathrm{p}, \mathrm{c})$ for some $\mathrm{c} \in[0,1)$. Assume the following.

(i) For any $\varepsilon>0$, there exists $\delta>0$ such that $\mathrm{p}(\mathrm{x}, \mathrm{y})<\varepsilon+\delta$ implies $\mathrm{d}(\mathrm{Tx}, \mathrm{Ty}) \leqslant \varepsilon$.

(ii) $\mathrm{p}(\mathrm{x}, \mathrm{y})>0$ implies $\mathrm{d}(\mathrm{T} x, \mathrm{Ty})<\mathrm{p}(\mathrm{x}, \mathrm{y})$.

Then $\mathrm{T}$ has a unique fixed point $z$. Moreover $\left\{\mathrm{T}^{\mathrm{n}} \mathrm{\chi}\right\}$ converges to $z$ for all $\mathrm{x} \in \mathrm{X}$.

Corollary 4.7. Let $(\mathrm{X}, \mathrm{d})$ be a complete metric space and let $\mathrm{T}$ be a continuous mapping on $\mathrm{X}$. Let $\mathrm{p}$ be a function from $\mathrm{X} \times \mathrm{X}$ into $[0, \infty)$ satisfying (P1:p). Assume (i) and (ii) of Corollary 4.6. Then $\mathrm{T}$ has a unique fixed point $z$. Moreover $\left\{\mathrm{T}^{\mathrm{n}} \mathrm{x}\right\}$ converges to $z$ for all $\mathrm{x} \in \mathrm{X}$. 


\section{Deduced theorems}

In this section, we state some theorems, which can be deduced by Theorems 3.1 and 3.2. The following are known results.

Theorem $5.1([3,7,10,11])$. Let $(\mathrm{X}, \mathrm{d})$ be a complete metric space and let $\mathrm{T}$ be a mapping on $\mathrm{X}$. Assume the following.

(i) For any $\varepsilon>0$, there exists $\delta>0$ such that $\mathrm{d}(\mathrm{x}, \mathrm{y})<\varepsilon+\delta$ implies $\mathrm{d}(\mathrm{T} x, \mathrm{Ty}) \leqslant \varepsilon$.

(ii) $x \neq y$ implies $\mathrm{d}(\mathrm{T} x, \mathrm{Ty})<\mathrm{d}(\mathrm{x}, \mathrm{y})$.

Then $\mathrm{T}$ has a unique fixed point $z$. Moreover $\left\{\mathrm{T}^{\mathrm{n}} \mathrm{x}\right\}$ converges to $z$ for all $\mathrm{x} \in \mathrm{X}$.

Proof. It is obvious that (ii) is equivalent to the following:

(ii') $d(x, y)>0$ implies $d(T x, T y)<d(x, y)$.

We obtain the desired result by Example 2.2 (i) and Corollary 4.6.

Theorem 5.2. Let $(\mathrm{X}, \mathrm{d})$ be a complete metric space and let $\mathrm{T}$ be a mapping on $\mathrm{X}$. Assume the following.

(i) For any $\varepsilon>0$, there exists $\delta>0$ such that $\mathrm{K}(\mathrm{x}, \mathrm{y})<\varepsilon+\delta$ implies $\mathrm{d}(\mathrm{T} x, \mathrm{Ty}) \leqslant \varepsilon$.

(ii) $x \neq y$ implies $\mathrm{d}(\mathrm{T} x, \mathrm{~T} y)<\mathrm{K}(\mathrm{x}, \mathrm{y})$.

Then $\mathrm{T}$ has a unique fixed point $z$. Moreover $\left\{\mathrm{T}^{\mathrm{n}} \mathrm{x}\right\}$ converges to $z$ for all $\mathrm{x} \in \mathrm{X}$.

Remark 5.3. The author does not know who first proved Theorem 5.2.

Proof. It is obvious that $\mathrm{K}(\mathrm{x}, \mathrm{y})=0 \Rightarrow \mathrm{x}=\mathrm{y}$ holds. So by Lemma 4.2, (ii) is equivalent to the following.

(ii') $K(x, y)>0$ implies $d(T x, T y)<K(x, y)$.

We obtain the desired result by Example 2.2 (ii) and Corollary 4.6.

Theorem 5.4 ([7, Theorem 2]). Let $(\mathrm{X}, \mathrm{d})$ be a complete metric space and let $\mathrm{T}$ be a continuous mapping on $\mathrm{X}$. Assume the following.

(i) For any $\varepsilon>0$, there exists $\delta>0$ such that $\mathrm{L}(\mathrm{x}, \mathrm{y})<\varepsilon+\delta$ implies $\mathrm{d}(\mathrm{T} x, \mathrm{Ty}) \leqslant \varepsilon$.

(ii) $\mathrm{L}(\mathrm{x}, \mathrm{y})>0$ implies $\mathrm{d}(\mathrm{T} x, \mathrm{Ty})<\mathrm{L}(\mathrm{x}, \mathrm{y})$.

Then $\mathrm{T}$ has a unique fixed point $z$. Moreover $\left\{\mathrm{T}^{\mathrm{n}} \mathrm{x}\right\}$ converges to $z$ for all $\mathrm{x} \in \mathrm{X}$.

Proof. We obtain the desired result by Example 2.2 (iii) and Corollary 4.7.

The following is a generalization of Theorems 1.1-1.3. See also [9].

Theorem 5.5. Let $(\mathrm{X}, \mathrm{d})$ be a complete metric space and let $\mathrm{T}$ be a mapping on $\mathrm{X}$. Define a function $\mathrm{p}$ from $\mathrm{X} \times \mathrm{X}$ into $[0, \infty)$ by

$$
p(x, y)=\max \left\{d(y, T y) \frac{1+d(x, T x)}{1+d(x, y)}, d(x, y)\right\} .
$$

Assume the following.

(i) For any $\varepsilon>0$, there exists $\delta>0$ such that $p(x, y)<\varepsilon+\delta$ implies $d(T x, T y) \leqslant \varepsilon$.

(ii) $x \neq y$ implies $\mathrm{d}(\mathrm{T} x, \mathrm{Ty})<\mathrm{p}(\mathrm{x}, \mathrm{y})$.

Then $\mathrm{T}$ has a unique fixed point $z$. Moreover $\left\{\mathrm{T}^{\mathrm{n}} \mathrm{x}\right\}$ converges to $z$ for all $\mathrm{x} \in \mathrm{X}$.

Proof. It is obvious that $p(x, y)=0 \Rightarrow x=y$ holds. So by Lemma 4.2, (ii) is equivalent to the following:

(ii') $p(x, y)>0$ implies $d(T x, T y)<p(x, y)$. 
We note that $\mathrm{p}$ is identical to $\mathrm{p}_{2}$ in Example 2.3. So the conclusion follows from Corollary 4.6.

In order to show that Theorem 5.5 is a generalization of Theorem 1.1, we prove the following.

Lemma 5.6. If all the assumptions of Theorem 1.1 hold, then all the assumptions of Theorem 5.5 hold.

Proof. We assume all the assumptions of Theorem 1.1. Let $p$ be as in Theorem 5.5 and define a function $q$ from $X \times X$ into $[0, \infty)$ by

$$
q(x, y)=\frac{1}{2}\left(d(y, T y) \frac{1+d(x, T x)}{1+d(x, y)}+d(x, y)\right) .
$$

It is obvious that $q \leqslant p$ holds. We note that if $q(x, y)=0$ holds, then $x=y$ holds and hence $d(T x, T y)=0$ holds. So by Lemma 2.4, the following holds:

(a) For any $\varepsilon>0$, there exists $\delta>0$ such that

$$
\mathrm{q}(x, y)<\varepsilon+\delta \text { implies } \mathrm{d}(\mathrm{Tx}, \mathrm{Ty})<\varepsilon .
$$

Since $q \leqslant p$ holds, we obtain (i) of Theorem 5.5. From (a), the following holds:

(b) $q(x, y)>0$ implies $d(T x, T y)<q(x, y)$.

Since $q \leqslant p$ holds, we obtain (ii') in the proof of Theorem 5.5, which is equivalent to (ii) of Theorem 5.5 .

We also obtain the following.

Theorem 5.7. Let $(\mathrm{X}, \mathrm{d})$ be a complete metric space and let $\mathrm{T}$ be a continuous mapping on $\mathrm{X}$. Define a function $\mathrm{p}$ from $\mathrm{X}^{(2)}$ into $[0, \infty)$ by

$$
p(x, y)=\max \left\{d(y, T y) \frac{1+d(x, T x)}{1+d(x, y)}, \frac{d(x, T x) d(y, T y)}{d(x, y)}, d(x, y)\right\} .
$$

Assume (i) and (ii) of Theorem 3.1. Then $\mathrm{T}$ has a unique fixed point $z$. Moreover $\left\{\mathrm{T}^{\mathrm{n}} \mathrm{x}\right\}$ converges to $z$ for all $\mathrm{x} \in \mathrm{X}$. Proof. We note that $\mathrm{p}$ is identical to $\mathrm{p}_{5}$ in Example 2.3. So the conclusion follows from Theorem 3.2.

We finally prove Theorem 2.1 in [13] by using Theorem 5.7. In other words, Theorem 5.7 is a generalization of Theorem 5.8. See also [5].

Theorem 5.8 ([13, Theorem 2.1]). Let $(\mathrm{X}, \mathrm{d})$ be a complete metric space and let $\mathrm{T}$ be a continuous mapping on $\mathrm{X}$. Define a function $\mathrm{q}$ from $\mathrm{X}^{(2)}$ into $[0, \infty)$ by

$$
q(x, y)=\frac{1}{3}\left(d(y, T y) \frac{1+d(x, T x)}{1+d(x, y)}+\frac{d(x, T x) d(y, T y)}{d(x, y)}+d(x, y)\right) .
$$

Assume that for any $\varepsilon>0$, there exists $\delta>0$ such that

$$
x \neq y, \varepsilon \leqslant q(x, y)<\varepsilon+\delta \text { imply } \mathrm{d}(\mathrm{Tx}, \mathrm{Ty})<\varepsilon .
$$

Then $T$ has a unique fixed point $z$. Moreover $\left\{\mathrm{T}^{\mathrm{n}} \mathrm{x}\right\}$ converges to $z$ for all $\mathrm{x} \in \mathrm{X}$.

Proof. Let $p$ be as in Theorem 5.7. Putting $p(x, x)=q(x, x)=1$, we extend the domains of $p$ and $q$ to $X \times X$. It is obvious that $q \leqslant p$ holds. We note that $q(x, y)=0$ cannot be possible. Thus, $q(x, y)=0$ implies $\mathrm{d}(\mathrm{Tx}, \mathrm{Ty})=0$. So by Lemma 2.4, the following holds:

(a) For any $\varepsilon>0$, there exists $\delta>0$ such that

$$
x \neq y, q(x, y)<\varepsilon+\delta \quad \text { imply } \quad d(T x, T y)<\varepsilon .
$$

Hence we obtain (i) of Theorem 3.1. From (a), the following holds:

(b) $x \neq y$ and $q(x, y)>0$ imply $d(T x, T y)<q(x, y)$.

Hence we obtain (ii) of Theorem 3.1. By Theorem 5.7, we obtain the desired result. 


\section{Acknowledgment}

The author is supported in part by JSPS KAKENHI Grant Number 16K05207 from Japan Society for the Promotion of Science.

\section{References}

[1] S. Banach, Sur les opérations dans les ensembles abstraits et leur application aux équations intégrales, Fund. Math., 3 (1922), 133-181. 1

[2] R. Caccioppoli, Un teorema generale sull'esistenza di elementi uniti in una transformazione funzionale, Rend. Accad. Lincei, 11 (1930), 794-799. 1

[3] L. Ćirić, A new fixed-point theorem for contractive mappings, Publ. Inst. Math. (Beograd), 30 (1981), 25-27. 5.1

[4] B. K. Dass, S. Gupta, An extension of Banach contraction principle through rational expression, Indian J. Pure Appl. Math., 6 (1975), 1455-1458. 1, 1.2

[5] A. N. Gupta, A. Saxena, A unique fixed point theorem in metric spaces, Math. Student, 52 (1984), 156-158. 5

[6] M. Hegedüs, T. Szilágyi, Equivalent conditions and a new fixed point theorem in the theory of contractive type mappings, Math. Japon., 25 (1980), 147-157. 4

[7] J. Jachymski, Equivalent conditions and the Meir-Keeler type theorems, J. Math. Anal. Appl., 194 (1995), 293-303. 5.1, 5.4

[8] J. Jachymski, Remarks on contractive conditions of integral type, Nonlinear Anal., 71 (2009), 1073-1081. 4

[9] E. Karapinar, W. Shatanawi, K. Taş, Fixed point theorem on partial metric spaces involving rational expressions, Miskolc Math. Notes, 14 (2013), 135-142. 5

[10] M. Kuczma, B. Choczewski, R. Ger, Iterative functional equations, Cambridge University Press, Cambridge, (1990). 5.1

[11] J. Matkowski, Fixed point theorems for contractive mappings in metric spaces, Časopis Pěst. Mat., 105 (1980), 341-344. 5.1

[12] A. Meir, E. Keeler, A theorem on contraction mappings, J. Math. Anal. Appl., 28 (1969), 326-329. 1, 1.3

[13] N. Redjel, A. Dehici, İ. M. Erhan, A fixed point theorem for Meir-Keeler type contraction via Gupta-Saxena expression, Fixed Point Theory Appl., 2015 (2015), 9 pages. 5, 5.8

[14] S. Samet, C. Vetro, H. Yazidi, A fixed point theorem for a Meir-Keeler type contraction through rational expression, J. Nonlinear Sci. Appl., 6 (2013), 162-169. 1.1

[15] T. Suzuki, Discussion of several contractions by Jachymski's approach, Fixed Point Theory Appl., 2016 (2016), 11 pages. 4 